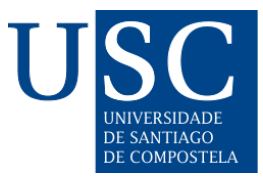

\title{
AUGE ELECTORAL DE LA EXTREMA DERECHA ESPAÑOLA. ANÁLISIS DE LA IRRUPCIÓN Y EVOLUCIÓN DEL VOTO DE VOX (2018-2019)
}

Electoral rise of the Spanish far-right. Analysis of the emergence and evolution of the VOX vote (2018-2019)

\author{
Gemma Vicente Iglesias ${ }^{1, a}$, Gonzalo Rodríguez Martínez ${ }^{2, b}$, Álvaro González Gómez ${ }^{3, c}$ \\ ${ }^{1}$ Universidade de Santiago de Compostela, España \\ ${ }^{2}$ Universidade de Santiago de Compostela, España \\ ${ }^{3}$ Universidade de Santiago de Compostela, España \\ a gemma.vicente@rai.usc.es b gonzalo.rmartinez@usc.es ca.gonzalez.gomez@usc.es
}

\section{Resumen}

El presente trabajo de investigación pretende ser una recapitulación pormenorizada desde los postulados clásicos de la Ciencia Política cuya finalidad será la de ordenar y explicar el auge y consolidación del partido de extrema derecha VOX dentro del sistema político y de partidos en España. Para ello, realizaremos un análisis que constará de tres prismas diferenciados, a saber, un primero que se pregunta acerca de las determinaciones que posibilitan el nacimiento y crecimiento del partido basado en una revisión bibliográfica. Un segundo prisma cuyo objetivo será el de describir a aquellos ciudadanos que optan por votar a VOX diseñando perfiles de votantes y, por último, un tercer espacio dedicado a explicar la composición del voto al partido de extrema derecha español a través de modelos de regresión logística binaria. De esta forma, tratamos de responder a tres preguntas de investigación: ¿Por qué surge VOX?, ¿Quién vota a VOX? Y ¿Cuáles son los elementos explicativos del voto a VOX?

Palabras clave: VOX; extrema derecha; Estructura de Oportunidad Política; perfil de votante; análisis electoral; comportamiento electoral.

\begin{abstract}
This research work aims to be a detailed recapitulation from the classic postulates of Political Science whose purpose will be to order and explain the rise and consolidation of the extreme right party VOX within the political and party system of Spain. To do this, we will carry out an analysis that will consist of three differentiated prisms, namely, a first that asks about the determinations that allow the birth and growth of the party based on a bibliographic review. A second prism whose objective will be to describe those citizens who choose to vote for VOX by designing voter profiles, and finally, a third space dedicated to explaining the composition of the vote for the Spanish far-right party through binary logistic regression models. In this way, we try to answer three questions: Why does VOX arise? Who votes for VOX? And why do they vote for VOX?
\end{abstract}


Keywords: VOX; far-right; political opportunity structure; voter profile; electoral analysis; electoral behaviour.

\section{INTRODUCCIÓN}

La democracia española (junto a la portuguesa), se había diferenciado hasta hace poco del resto de sus homólogas europeas, por la ausencia en su sistema de partidos de una opción política relevante de extrema derecha. Esta excepcionalidad tocó a su fin recientemente. En el caso concreto de España, la entrada y auge del partido político VOX en el escenario político español, es lo que ha provocado este cambio. Específicamente, luego de que hubiesen transcurrido varios años desde su reciente fundación, VOX inició en 2018 su salida de la marginalidad política en la que se encontraba. Pasó en un año de ser una formación política residual que solo contaba con una minúscula representación municipal, a acceder a todos los niveles del sistema política español; llegando en la repetición electoral de las elecciones generales de noviembre de 2019 a ser el tercer partido con mayor representación parlamentaria.

Este rápido auge de un partido de extrema derecha en España supone un fenómeno de interés tanto para la academia, (sobre todo por tratarse de un caso que se puede encuadrar dentro del tema de la extrema derecha, actualmente de relevancia) como para la sociedad en general (ante la entrada de un nuevo partido relevante en la escena política y el debate suscitado alrededor de cómo se debería categorizar a este nuevo actor). Respondiendo a este interés, en este artículo, pretendemos analizar la evolución y el auge de VOX. Para ello, abordaremos este estudio desde una aproximación tanto cualitativa como cuantitativa.

El artículo se estructura de la siguiente manera. Antes de nada, se presentarán los objetivos y la metodología de esta investigación. A continuación, analizaremos los resultados obtenidos de nuestro estudio, desde una aproximación cualitativa. En concreto, primero se presentará una breve síntesis de la historia reciente de la extrema derecha española (la familia ideológica en la que se encuentra VOX) para, a continuación, tratar de identificar cuál es la Estructura de Oportunidad Política que permitió el auge de VOX. Por último, se presentará una breve reflexión sobre qué categoría se debería usar para identificar a esta formación: si se trata de un partido de extrema derecha o de derecha radical populista. Por otro lado, desde una aproximación cuantitativa, y utilizando datos propios del Equipo de Investigaciones Políticas de la Universidad de Santiago de Compostela (EIP-USC), se definirá el perfil sociopolítico de los votantes de VOX (dando cuenta de una amplia gama de variables de relevancia y su evolución más reciente), para posteriormente analizar la importancia/influencia de las variables clásicas en la explicación de voto a este partido. Por último, presentaremos las conclusiones de nuestra investigación.

\section{OBJETIVOS Y METODOLOGÍA DE LA INVESTIGACIÓN}

De cara a la realización del trabajo que, previamente se ha presentado, planteamos tres objetivos fundamentales: 1) Describir la evolución del voto a VOX; 2) Definir el perfil sociopolítico de los votantes de dicho partido; y 3) Analizar cuáles son los elementos clásicos que explican el voto a VOX. 
Tabla 1. Datos técnicos de los estudios demoscópicos utilizados en el análisis

Nombre estudio

Datos técnicos

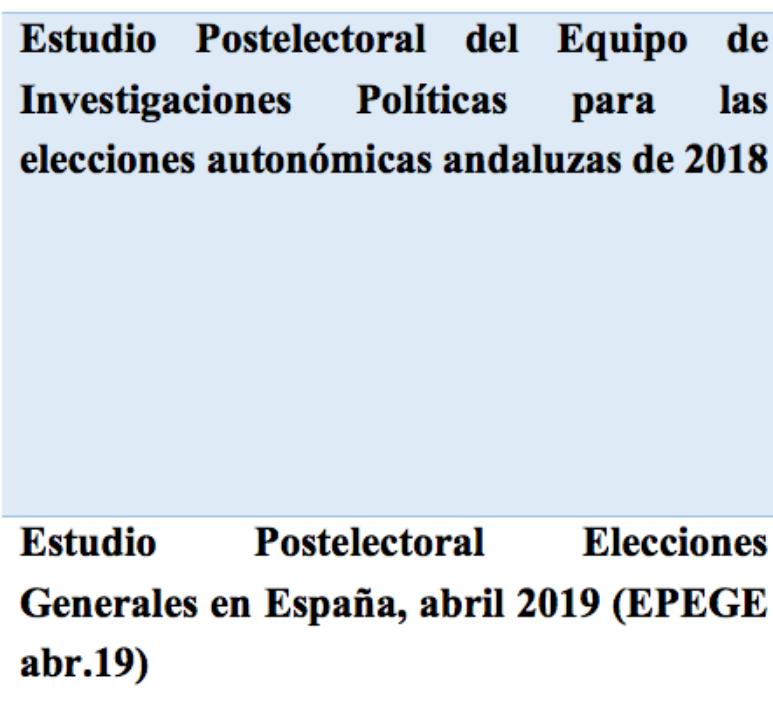

Estudio postelectoral Elecciones
Generales en España, noviembre 2019
(EPEGE, nov.19)

Fechas: 12 de junio -12 de julio de 2019.

Universo: población mayor de 18 años, residente en

España.

Tamaño muestral: 1000 .

Supuestos: $\mathrm{p}=\mathrm{q}$.

Error asociado: $\pm 3,1 \%$.

Afijación: proporcional (cuotas de sexo y edad).

Administración: telefónicamente mediante el sistema

CATI.

\begin{tabular}{|l|l|}
\hline Estudio postelectoral Elecciones & Fechas: 14 de enero -22 de febrero de 2020. \\
Generales en España, noviembre 2019 & Universo: población mayor de 18 años, residente en \\
España. & Tamaño muestral: 1000. \\
& Supuestos: $\mathrm{p}=\mathrm{q}$ \\
& Error asociado: $\pm 3,1 \%$. \\
& Afijación: proporcional (cuotas de sexo, edad y \\
& provincia). \\
& Administración: telefónicamente mediante el sistema \\
& CATI.
\end{tabular}

Fuente: elaboración propia a partir del Estudio Postelectoral del EIP para las elecciones andaluzas de 2018, el Estudio Postelectoral de las Elecciones Generales en España de abril y noviembre de 2019 (EPEGE, abril y noviembre 2019)

Para la consecución de estos objetivos, optaremos por un enfoque metodológico mixto. En primer lugar, llevaremos a cabo un análisis longitudinal que abarca desde las elecciones generales de 2015, hasta las celebradas en noviembre de 2019. Dentro de ese período, también incluiremos como elemento de análisis, las elecciones autonómicas de Andalucía de 2018 por ser el primer gran hito en la presencia institucional de esta formación. En segundo lugar, realizaremos un enfoque metodológico descriptivo a través del cual, estudiaremos cómo es el votante de VOX, qué lo define, de dónde viene y, cuáles han sido los motivos que lo han llevado a votar a dicho partido. Por último, llevaremos a cabo un análisis cuantitativo multivariante mediante regresión logística binaria de las elecciones autonómicas de Andalucía en 2018 y, las generales de abril y noviembre de 2019. Nuestra 
intención con este análisis radica en conocer cuáles son los elementos clásicos que explicarían el voto a VOX. Para llevar a cabo el análisis, nos serviremos de los datos recogidos en los estudios demoscópicos realizados por el EIP-USC, en los años mencionados. El lector puede comprobar las características técnicas de estos trabajos en la tabla 1.

\section{LA EXTREMA DERECHA EN ESPAÑA: EL CASO DE VOX}

\subsection{Breve historia sobre el surgimiento y evolución de VOX}

Hasta el auge de VOX, la extrema derecha española, se había destacado por su marginalidad y fragmentación interna. Desde Fuerza Nueva, formación por antonomasia del bunker tardofranquista, pasando por Democracia Nacional y su proyecto más similar a sus homólogos europeos (destacando su postura anti-inmigración), estas formaciones no habían sido capaces de lograr ningún éxito electoral (Alonso y Rovira, 2014; Casals, 2000). Como mucho, Plataforma per Catalunya, consiguió buenos resultados a nivel local centrándose en la oposición a la inmigración (sobre todo a la de carácter magrebí) y a los partidos políticos tradicionales (al estilo de la derecha radical populista) pero, evitando, (de forma excepcional) posicionarse en el eje nacionalista y asociarse con la extrema derecha tradicional española (Hernández-Carr, 2011).

En este contexto se hallaba la extrema derecha española cuando apareció en la escena VOX (Ferreira, 2019; Turnbull-Dugarte, 2019; Arroyo, 2020; Jaráiz, Rivera y Castro, 2021; López y Colomé, 2021; López-López y González, 2021). Este partido se fundó en 2013 como una escisión del Partido Popular (PP), motivada por una supuesta moderación, girando hacia el centro y convirtiéndose en la "derechita cobarde". Ante ello y, bajo el liderazgo inicial de Vidal-Quadras (antiguo eurodiputado del PP), se postulaban como defensores del "centroderecha nacional". Después de los comicios europeos de 2014 (en los que se quedó a unos miles de votos de conseguir un eurodiputado) y, el relevo del liderazgo con el ascenso interno de Santiago Abascal (otro destacado exmiembro del PP), se inicia una etapa en la historia de VOX caracterizada por el empeoramiento tanto de sus resultados electorales, (tabla 2) como de su prospección demoscópica (Tabla 3), no llegando a superar durante esta etapa ni el $1 \%$ de votos ni el $0,2 \%$ en intención de voto. Durante estos mismos años se inició su acercamiento hacia la extrema derecha europea, culminando este proceso en su asistencia a la Cumbre Libertad para Europa, celebrada en enero de 2017 en Coblenza, Alemania. 
Tabla 2. Resultados electorales de VOX (2014-2021)

\begin{tabular}{|c|c|c|c|c|}
\hline Año & Elecciones & Votos & $\%$ Voto & $\mathbf{N}^{0}$ escaños \\
\hline 2014 & Europeas & 244.929 & $1,56 \%$ & 0 \\
\hline \multirow{4}{*}{2015} & Municipales & 64.385 & $0,29 \%$ & 22 \\
\hline & Autonómicas Andalucía & 18.017 & $0,45 \%$ & 0 \\
\hline & Autonómicas (10 ccaa.) & 75.486 & $0,62 \%$ & 0 \\
\hline & Generales & 58.114 & $0,2 \%$ & 0 \\
\hline \multirow{2}{*}{2016} & Generales & 47.182 & $0,2 \%$ & 0 \\
\hline & Autonómicas País Vasco & 774 & $0,07 \%$ & 0 \\
\hline 2018 & Autonómicas Andalucía & 395.978 & $10,97 \%$ & 12 \\
\hline \multirow{6}{*}{2019} & Autonómicas Com. Valenciana & 278.947 & $10,44 \%$ & 10 \\
\hline & Generales (abril) & 2.664 .325 & $10,26 \%$ & 24 \\
\hline & Europeas & 1.388 .681 & $6,2 \%$ & 3 \\
\hline & Autonómicas (14 ccaa.) & 684.312 & $6,67 \%$ & 35 \\
\hline & Municipales & 659.736 & $2,9 \%$ & 530 \\
\hline & Generales (noviembre) & 3.656 .979 & $15,09 \%$ & 52 \\
\hline \multirow{2}{*}{2020} & Autonómicas País Vasco & 17.569 & $1,96 \%$ & 1 \\
\hline & Autonómicas Galicia & 26.797 & $2,05 \%$ & 0 \\
\hline \multirow{2}{*}{2021} & Autonómicas Cataluña & 218.121 & $7,67 \%$ & 11 \\
\hline & Autonómicas Madrid & 330.660 & $9,13 \%$ & 13 \\
\hline
\end{tabular}

Fuente: Elaboración propia a partir de Ferreira (2019) y datos del Ministerio del Interior y de los gobiernos autonómicos gallego, vasco, catalán y madrileño (Xunta de Galicia, 2020; Gobierno vasco, 2020; Asamblea de Madrid, 2021).

Con todo, esta etapa tocó a su fin en 2018, al iniciarse definitivamente el surgimiento de VOX, convirtiéndose entonces en un partido relevante en términos demoscópicos y electorales. Precediendo a esto, es de destacar como en el barómetro de octubre de 2017 (tabla 2) ya había conseguido alcanzar el 0,2\% de intención directa de voto, justo cuando se estaba desarrollando el referéndum independentista catalán. Sin embargo, el surgimiento y auge definitivo no se produciría hasta un año después, iniciándose al principio en el barómetro de julio un alza demoscópica que se prolongaría a lo largo del resto del año y, que cristalizaría electoralmente en los comicios andaluces de diciembre, consiguiendo el 10,97\% de los votos y 12 escaños (tabla 1).

Las elecciones andaluzas, darían pie al "año electoral" de 2019, en el que se celebraron elecciones municipales, autonómicas, generales (incluida la repetición de noviembre) y europeas A lo largo de estas convocatorias VOX accedió a los distintos niveles del sistema político español, aunque con suerte heterogénea, alcanzando su mejor resultado en las generales de noviembre y el peor en las municipales de dicho año (tabla 2). 
Tabla 3. Evolución demoscópica de VOX en elecciones generales y europeas (2014-2019)

\begin{tabular}{|c|c|c|c|c|c|}
\hline Año & Fecha & Estudio & Voto directo & Voto+Simpatía & $\begin{array}{c}\text { Voto } \\
\text { estimado }\end{array}$ \\
\hline 2014 & $07-a b r-14$ & Pre-EUR 2014 (3022) & 0,3 & 0,4 & 0,5 \\
\hline \multirow{3}{*}{2015} & $01-a b r-15$ & Bar. Abr-2015 (3080) & 0,1 & & \\
\hline & 01-jul-15 & Bar. Jul-2015 (3104) & 0,1 & & \\
\hline & 27 -oct-15 & Pre-ESP 2015 (3117) & 0,1 & & \\
\hline \multirow{3}{*}{2016} & 02-ene-16 & Bar. Ene-2016 (3124) & 0,1 & & \\
\hline & 04-may-16 & Pre-ESP 2016 (3141) & 0 & 0 & \\
\hline & 01 -oct-16 & Bar. Oct-2016 (3156) & 0,1 & & \\
\hline \multirow{2}{*}{2017} & 02-ene-17 & Bar. Ene-2017 (3164) & 0 & & \\
\hline & 02 -oct-17 & Bar. Oct-2017 (3191) & 0,2 & & \\
\hline \multirow{3}{*}{2018} & 01-jul-18 & Bar. Jul-2018 (3219) & 0,5 & 0,5 & \\
\hline & 01-sep-18 & Bar. Sep-2018 (3223) & 0,9 & 1 & 1,4 \\
\hline & 01-oct-18 & Bar. Oct-2018 (3226) & 1 & 1 & 1,3 \\
\hline \multirow{5}{*}{2019} & 01-ene-19 & Bar. Ene-2019 (3238) & 4,5 & 4,7 & 6,5 \\
\hline & 01-mar-19 & Pre-ESP 2019 (3242) & 3,8 & 4,4 & 11,9 \\
\hline & 01-may-19 & Bar. May-2019 (3247) & 4,4 & 4,6 & 5,3 \\
\hline & 21-sep-19 & Pre-ESP 2019 (3263) & 4 & 4,6 & 7,9 \\
\hline & 28-oct-19 & Bar. Nov-2019 (3267) & 7,3 & 8,1 & \\
\hline
\end{tabular}

Fuente: Centro de Investigaciones Sociológicas (CIS)

Por último, en el contexto marcado por la pandemia, la oposición de VOX al gobierno estatal de coalición progresista (que incluyó la gestión de esta crisis sanitaria en el repertorio de agravios) llegó a su cenit con la presentación de una moción de censura al gobierno (finalmente derrotada) en octubre de 2020, con la cual VOX se pretendió erigir como líder de la oposición y, por lo tanto, de la derecha.

\subsection{Análisis del auge de VOX}

Planteada esta narración cronológica, seguiremos a Tarrow (1994) y el ejemplo práctico de Lagares y Pereira (2015), al utilizar el concepto de Estructura de Oportunidad Política compuesto por los siguientes cuatro elementos ${ }^{1}$ para intentar aproximarnos a la identificación de las claves que permitieron el surgimiento de VOX años después de su fundación y de haberse presentado infructuosamente a varios comicios anteriormente (en concreto, en la segunda mitad del año 2018). 


\subsubsection{Apertura institucional al acceso a la participación}

El conjunto de elecciones entre diciembre de 2018 y noviembre de 2019 (tanto las ya programadas en su momento como las anticipadas) se convirtieron en una clara oportunidad para cualquier outsider al sistema de partidos para acceder a las instituciones representativas. La posibilidad de concurrir a varias elecciones en un espacio tan breve de tiempo se combinó, además, para el caso de VOX, con un escenario de alza demoscópica iniciado en julio de 2018 e impulsada por los buenos resultados electorales de las andaluzas de diciembre (tabla 2).

\subsubsection{Cambios de alineamientos políticos}

A la hora de considerar el lado de la demanda, varios autores ya habían señalado la presencia de una demanda concreta que podía posibilitar el surgimiento de una formación relevante de extrema derecha en España, bien resaltando las actitudes contrarias a la inmigración (Alonso y Rovira, 2014) o bien al nacionalismo catalán y el procés (Casals, 2017). A pesar de esto, la "excepcionalidad ibérica" en nuestro país se mantenía: ni había un partido relevante de extrema derecha, ni había visos de que lo hubiese haber en el futuro (Alonso y Rovira, 2014; Turnbull-Dugarte, 2019; Ferreira, 2019 y Turnbull-Dugarte et al., 2020).

¿A qué se debía esto? Por un lado, se señala (Casals, 2017), que se debe a la presencia de un partido capaz de abarcar a toda la derecha (PP) desde los elementos moderados hasta los extremistas. Por otro lado, se señala a mayores (Alonso y Rovira, 2014) que, junto a este elemento, también cobra importancia la importancia del cleavage territorial (nacionalista de Estado vs. nacionalista periférico) que impedía la articulación de una opción nativista (al dificultar la definición de un "nosotros" nativista) ${ }^{2}$ y un sistema electoral con los suficientes efectos desproporcionales como para dificultar la entrada de nuevos partidos (sin importar su carácter político-ideológico). En definitiva, la demanda de VOX ya existía, pero los factores de la oferta (de forma destacada, la presencia del PP) la contenía.

\subsubsection{Existencia de posibles alianzas políticas}

VOX se encontró con un escenario favorable para sus alianzas (una vez hubo iniciado su camino hacia la relevancia política) debido a que la derecha moderada (PP y Ciudadanos) lo aceptaron desde un primer momento como un posible miembro válido de coalición de gobierno. Con esta condición participó en los distintos pactos de gobiernos que estas formaciones forjaron a lo largo de 2018 y 2019 a nivel autonómico (en Andalucía, Madrid y Murcia) y municipal (como en Madrid, Granada, Zaragoza, Teruel, Palencia, Badajoz, Santander) (Pereira, Jaráiz y Cazorla, 2021).

Así, esta colaboración con VOX como "mal menor" ante la oposición frontal al bloque de izquierdas y nacionalista (algo que también se puede constatar en la manifestación de plaza Colón de febrero de 2019) estos pactos han permitido a VOX visibilizarse en el escenario público y explotar sus oportunidades y las debilidades de sus contrincantes de derecha para ganarles terreno, como se manifestó en su fallida moción de censura de 2020. 


\subsubsection{División entre y dentro élites}

Las élites en el contexto en el que hablamos (es decir, los partidos) se hallaban en el inicio del surgimiento de VOX en un estado de división perfecto para el auge de este partido.

Por un lado, respecto a la división entre partidos, nos encontramos por un lado (a un nivel general) un escenario político fragmentado después del fin del bipartidismo imperfecto que durante décadas había caracterizado al sistema político español, pasando a otro multipartidista más fragmentado, complejo e incierto (Montero y Fernández-Esquer, 2018). Además de esta fragmentación, la polarización (en concreto, la crispación) ya era desde el gobierno de Zapatero una marca característica de este escenario (Arroyo, 2020). Quizás más determinante fue la división entre los partidos de derecha moderada (PP y Ciudadanos) los cuales, insertos en una competencia constante por el electorado de centroderecha, han dejado abierto un espacio que ha sabido aprovechar VOX, al igual que ocurre con sus homólogos en el resto de Europa.

Sin embargo, lo verdaderamente determinante a nuestro parecer fue la división dentro de los partidos, en concreto del partido que ya se ha señalado anteriormente que imposibilitaba el auge electoral de la extrema derecha española: el PP. La coincidencia temporal es perfecta entre el inicio del ascenso demoscópico de VOX y la crisis interna abierta en el PP tras perder el gobierno central por la triunfante moción de censura de mayo de 2018 y el subsiguiente inicio de un conflictivo proceso congresual, en el que Casado sólo logró la victoria en la segunda fase después de pactar con el resto de las candidaturas perdedoras en la primera fase.

\subsubsection{Breve conclusión}

Recapitulando, el escenario político español de la segunda mitad de 2018 planteaba una Estructura de Oportunidad Política perfecta para el salto hacia la relevancia política de VOX.

De primeras, el que en el medio plazo se presentase un año electoral intenso en medio de la división del resto de partidos relevantes ya sugería una oportunidad mínima que explotar. Pero las consecuencias de la moción de censura de mayo ampliaron las expectativas de VOX, al encontrarse con que la principal barrera que imposibilitaba la cristalización electoral de su demanda electoral (es decir, el PP) se hallaba en un momento de destacada debilidad. Esta falta del "dique de contención del PP" se amplió cuando, a nada más que VOX consiguió cristalizar electoralmente su demanda electoral en Andalucía, se inició una política de pactos con PP y Ciudadanos. Si a esto le sumamos la presencia de un gobierno central de cuño político opuesto contra el que dirigir una oposición frontal, lo que tenemos es una oportunidad política perfecta.

\subsection{VOX: ¿extrema derecha europea?}

A continuación, queremos brevemente considerar dos preguntas. Primero, ¿por qué utilizar la categoría de extrema derecha para hablar de VOX? Segundo, ¿Cuáles son las similitudes entre VOX y sus homólogos europeos?

Empezando por el debate semántico, nos encontramos principalmente con el dilema de usar la categoría de derecha radical populista o la de extrema derecha. Por un lado, Mudde (2007 y 2019) señala que es preciso distinguir entre la extrema derecha tradicional (correspondiente a la primera 
y segunda ola) y la derecha radical populista, debido principalmente a que las olas posteriores de la extrema derecha asumieron el marco legal democrático, dejando de considerar la instauración de un régimen de corte autoritario o el uso de la violencia para fines políticos. Sin embargo, consideramos que, en la línea de Jaráiz, Rivera y Castro (2021), esta división se solapa demasiado con la de nuevos y viejos partidos de extrema derecha. Es decir, lo que Mudde identifica como "derecha radical populista" no sería más que la "nueva" extrema derecha que, asumiendo el cambio de escenario producido a mediados del siglo pasado, se adapta al escenario democrático (tendiendo a operar dentro de su sistema político) y postindustrial (adecuándose a las necesidades y temas que este produce). En este sentido, es de destacar, aunque sea a un nivel meramente descriptivo, que el $85 \%$ de los votantes de VOX en las elecciones andaluzas declaran ubicarse ideológicamente en posiciones comprendidas entre el 8 y 10 en una escala comprendida entre el 1 (extrema izquierda) y el 10 (extrema derecha) (Montabes y Cazorla, 2021).

Pasando al debate comparativo, consideremos primero que elementos son los que caracterizan a la extrema derecha europea. Algunos factores que la caracterizarían, por el lado de la oferta, serían cuestiones como la ideología o el liderazgo en estos partidos. Mientras que, por el lado de la demanda, destaca el nativismo o actitudes antiinmigración (Arzheimer, 2018; Lagares y Castro, 2021; Lubbers y Scheepers, 2001; Lubbers et al., 2002; Van der Brug et al., 2005), antiglobalización (Betz, 1994; Rydgren, 2006; Zaslove, 2008) o anti-establishment (Norris, 2018). Por otra parte, ciertos autores (Betz, 1994, 1998; Gaus, 2004) identifican en las características sociodemográficas los patrones de apoyo a estos partidos cuando se dan contextos de estrés y cambios cíclicos macroeconómicos, como el de la crisis económica iniciada en 2007 (Magalhaes, 2014). En este sentido, un factor sociodemográfico destacado sería el género (Betz, 1994; Lubbers, 2002; Montabes y Cazorla, 2021), en el sentido de presentar un perfil masculino más que femenino. Otro sería la edad, bien tendiendo su apoyo a provenir más de los más jóvenes (Teperoglou et al., 2016; Stockemer, 2017) o, de los más mayores (Rooduijn, 2017; Ford y Goodwin, 2014). El nivel de estudios sería por su parte un factor en el sentido de una mayor tendencia a apoyar a estos partidos entre los niveles formativos menores y, por lo tanto, de menor cualificación (Warwick, 1998 y Weakliem, 2002). Por último, respecto a la clase social (Kitschelt (1995), tanto aquellos trabajadores de sectores ajenos a la manufacturación y con cierta autonomía laboral, como votantes de la clase trabajadora manufacturera y artesanos, comerciantes o pequeños empresarios, pueden llegar a desarrollar preferencias autoritarias.

Entrando en el caso particular de VOX, la literatura existente, destaca tanto continuidades como discontinuidades respecto a sus homólogos europeos. De esta forma, teniendo similitudes ideológicas (nativismo, autoritarismo, aceptación del sistema democrático) y de perfil de votante, lo cierto es que VOX se diferencia por su postura más nacionalista que populista y su clara ubicación conservadora en lo social y, liberal en lo económico, además de un perfil sociológico más burgués (Ferreira, 2019; Turnbull-Dugarte et al., 2020). En esta línea, mientras que algunos autores destacan la importancia de las actitudes antiimigración (Castro y Mo, 2020), otros destacan la importancia del conflicto territorial y del nacionalismo español (Turnbull-Dugarte ,2019; TurnbullDugarte et al., 2020; Arroyo, 2020) 


\section{EVOLUCIÓN DEL VOTANTE DE VOX (2018-2019): DEL 2-D ANDALUZ A LA REPETICIÓN DEL 10-N.}

En esta sección del trabajo, elaboraremos un pequeño análisis sobre las particularidades que permiten caracterizar a los votantes de VOX.

\section{1 ¿Qué define al votante de VOX?}

Analizando los datos de las tres elecciones (tabla 4), observamos como el votante de VOX es un hombre, cuya edad oscila entre los 46 y los 50 años y en situación laboral activa. Respecto a su situación económica, ha mejorado desde 2018, al igual que sus estudios, donde primaban los estudios secundarios frente a los universitarios en 2019.

En cuanto a sus principales preocupaciones (gráfico 1), el desempleo destaca por ser el principal problema en las tres elecciones. Le sigue la clase política, la inmigración, la sanidad o la educación y la economía.

Acerca de su sentimiento nacionalista, observamos como los porcentajes han ido evolucionando en apenas un año (gráfico 2). Esto último resulta muy llamativo, pues desde las elecciones autonómicas de Andalucía, el porcentaje de encuestados que se sentía únicamente español ha aumentado en más de un $20 \%$. 
Tabla 4. Características sociodemográficas

\begin{tabular}{|c|c|c|c|}
\hline & ANDALUCÍA & ABRIL 2019 & NOVIEMBRE 2019 \\
\hline Sexo & Hombre $(51,1 \%)$ & Hombre $(75 \%)$ & Hombre $(58.9 \%)$ \\
\hline Edad (media) & 49 años & 46 años & 50 años \\
\hline Religión & $\begin{array}{c}\text { Católico no } \\
\text { practicante }(37,8 \%)\end{array}$ & $\begin{array}{c}\text { Católico poco } \\
\text { practicante }(46,4 \%)\end{array}$ & $\begin{array}{c}\text { Católico poco } \\
\text { practicante }(38,2 \%)\end{array}$ \\
\hline Situación laboral & Trabajador $(55,6 \%)$ & Trabajador $(46,4 \%)$ & Trabajador $(60,2 \%)$ \\
\hline Nivel de estudios & $\begin{array}{c}\text { Estudios secundarios } \\
(26,7 \%)\end{array}$ & $\begin{array}{c}\text { Estudios de grado } \\
\text { superior }(42,9 \%)\end{array}$ & $\begin{array}{c}\text { Estudios universitarios } \\
(26,7 \%)\end{array}$ \\
\hline Nivel de ingresos & $\begin{array}{c}1201-1800 € / \text { mes } \\
(34,9 \%)\end{array}$ & $\begin{array}{c}1201-1800 € / \text { mes } \\
(32,1 \%) \\
1801-2400 € / \text { mes } \\
(32,1 \%)\end{array}$ & $\begin{array}{c}1801-2400 € / \text { mes } \\
(27,6 \%)\end{array}$ \\
\hline Situación económica (0-10) & 5,36 & 5,89 & 6,07 \\
\hline $\begin{array}{l}\text { Nivel de felicidad } \\
\text { expresada }\end{array}$ & 8,07 & 7,25 & 7,59 \\
\hline
\end{tabular}

Fuente: Elaboración propia a partir del Estudio poselectoral del Equipo de Investigaciones Políticas para las elecciones autonómicas andaluzas 2018, del Estudio Postelectoral Elecciones Generales en España, abril 2019 (EPEGE abr.19) y del Estudio Postelectoral Elecciones Generales en España, noviembre 2019 (EPEGE nov.19). 
Gráfico 1. Principales problemas de España/Andalucía según votantes VOX

\author{
- Desempleo \\ - Clase política
}

GENERALES NOVIEMBRE 2019

GENERALES ABRIL 2019

ANDALUCÍA 2018

GENERALES ABRIL 2019

\author{
- Inmigración \\ a Sanidad \\ - Educación y enseñanza $₫$ Marcha de la economía
}

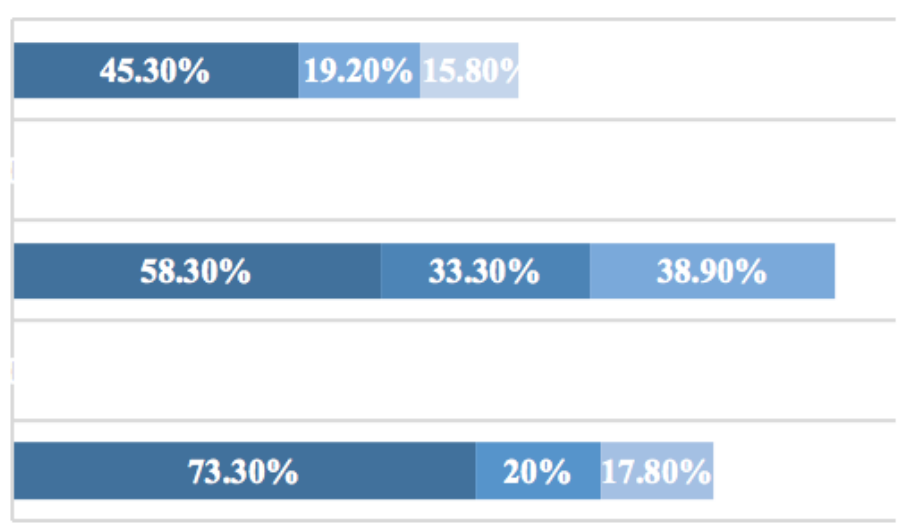

Fuente: Elaboración propia a partir del Estudio poselectoral del Equipo de Investigaciones Políticas para las elecciones autonómicas andaluzas 2018, del Estudio Postelectoral Elecciones Generales en España, abril 2019 (EPEGE abr.19) y del Estudio Postelectoral Elecciones Generales en España, noviembre 2019 (EPEGE nov.19).

Siguiendo con las variables de sentimiento de pertenencia, nos encontramos con la escala de autoubicación y ubicación de partidos en la escala nacionalista (gráfico 3) y, la escala nacionalista española (gráfico 4).

Gráfico 2. Pregunta Linz-Moreno

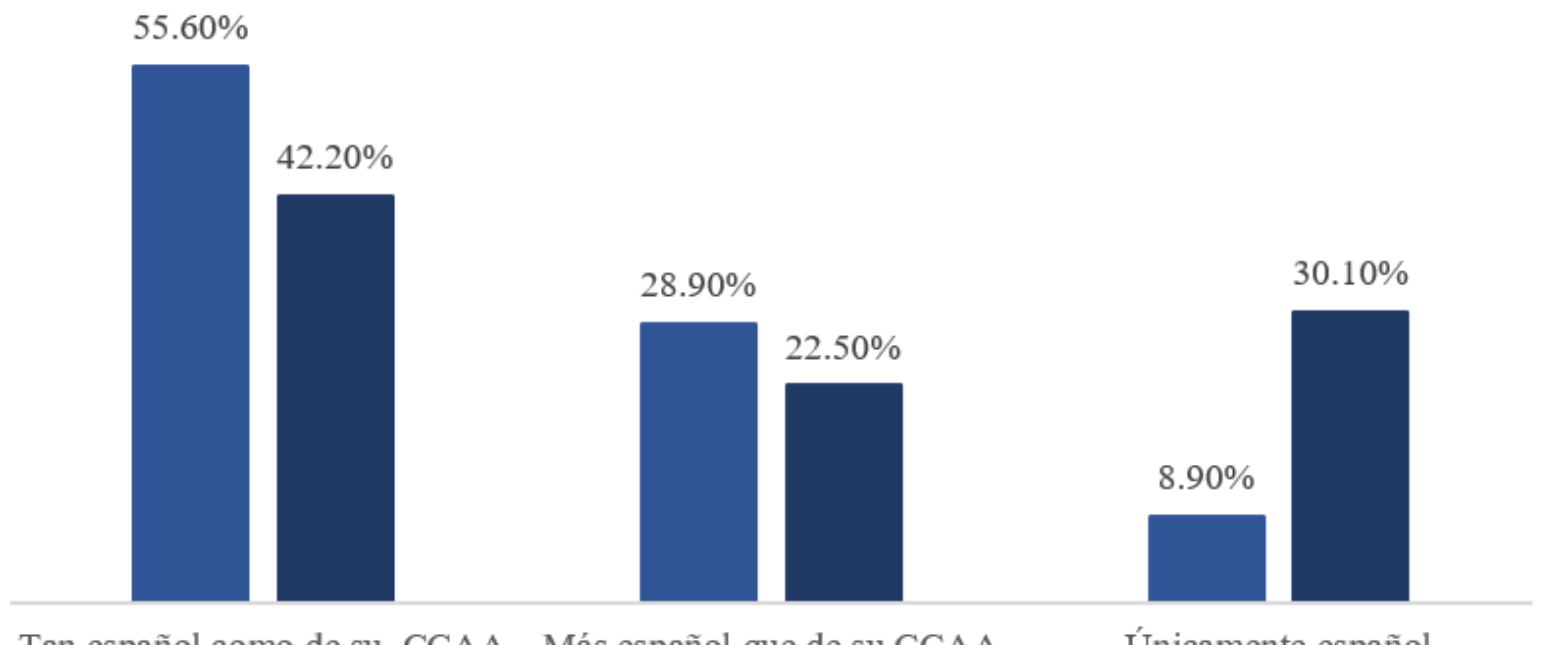

Tan español como de su CCAA Más español que de su CCAA Únicamente español

\section{Elecciones Andalucía de 2018 Elecciones Generales noviembre de 2019}

Fuente: Elaboración propia a partir del Estudio poselectoral del Equipo de Investigaciones Políticas para las elecciones autonómicas andaluzas 2018, del Estudio Postelectoral Elecciones Generales en España, abril 2019 (EPEGE abr.19) y del Estudio Postelectoral Elecciones Generales en España, noviembre 2019 (EPEGE nov.19).

Así pues, en el gráfico 3 se muestra una comparativa entre la autoubicación del propio votante en la escala nacionalista y, como estos ubican a VOX y al PP. Si comparamos las tres variables, 
observamos como en 2018 los valores eran muy parejos en los tres casos. Sin embargo, en abril de 2019 la ubicación de VOX y la autoubicación del votante se dispararon, llegando a 7,92 y 6,70 respectivamente, mientras que el PP se mantenía en un 5,73. En noviembre de 2019, la situación vuelve a cambiar y VOX y el Partido Popular vuelven a aproximar posturas, con un 3,64 y 3,46 respectivamente. En el caso de la autoubicación, los votantes bajaron de un 6,70 en abril, a un 5,34 en noviembre.

Mientras que en el caso de la escala de autoubicación nacionalista en noviembre, los votantes ubicaron al PP y VOX en valores cercanos al 3, mientras que, en la escala de ubicación nacionalista española (gráfico 4), estos los alzan hasta un 6,57 en el caso del PP y un 8,97 en el caso de VOX. Respecto a los líderes, los valores son muy similares a los de los partidos, situando a Casado en un 6,34 y a Abascal en un 8,96. En cuanto a la autoubicación, al igual que sucedió en los casos anteriores, el valor es más elevado en la escala de autoubicación nacionalista española que en la escala de autoubicación nacionalista, situándose en un 8,89.

Gráfico 3. Escala de ubicación nacionalista $(0-10)^{3}$

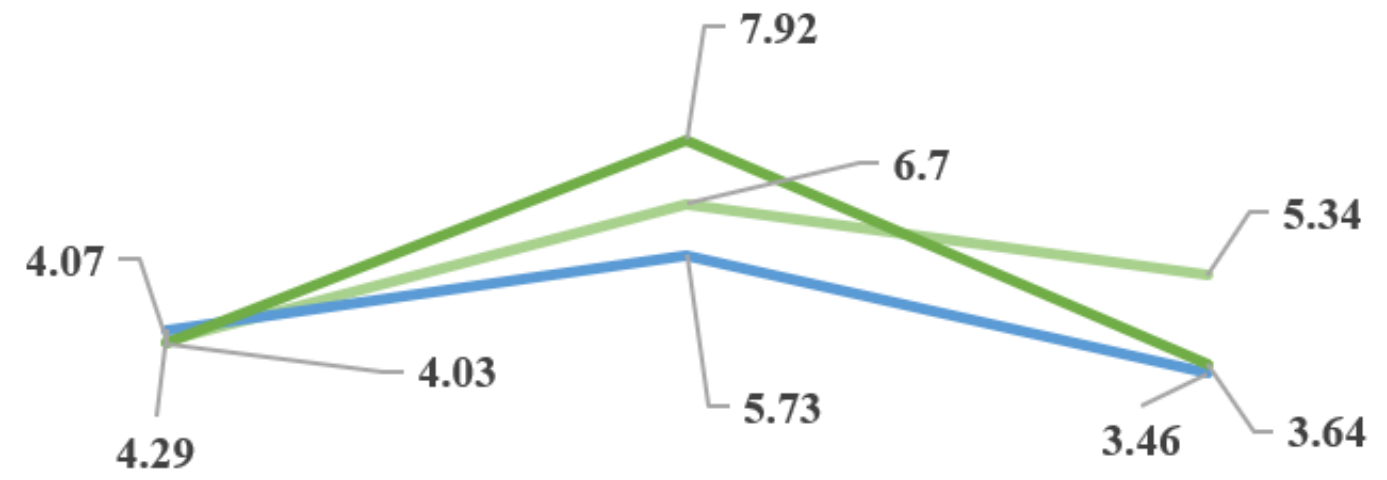

Andalucía 2018

Generales abril 2019

Generales noviembre 2019

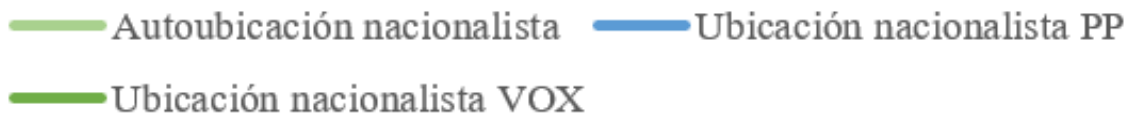

Fuente: Elaboración propia a partir del Estudio poselectoral del Equipo de Investigaciones Políticas para las elecciones autonómicas andaluzas 2018, del Estudio Postelectoral Elecciones Generales en España, abril 2019 (EPEGE abr.19) y del Estudio Postelectoral Elecciones Generales en España, noviembre 2019 (EPEGE nov.19). 
Gráfico 4. Escala de ubicación nacionalista española (0-10)

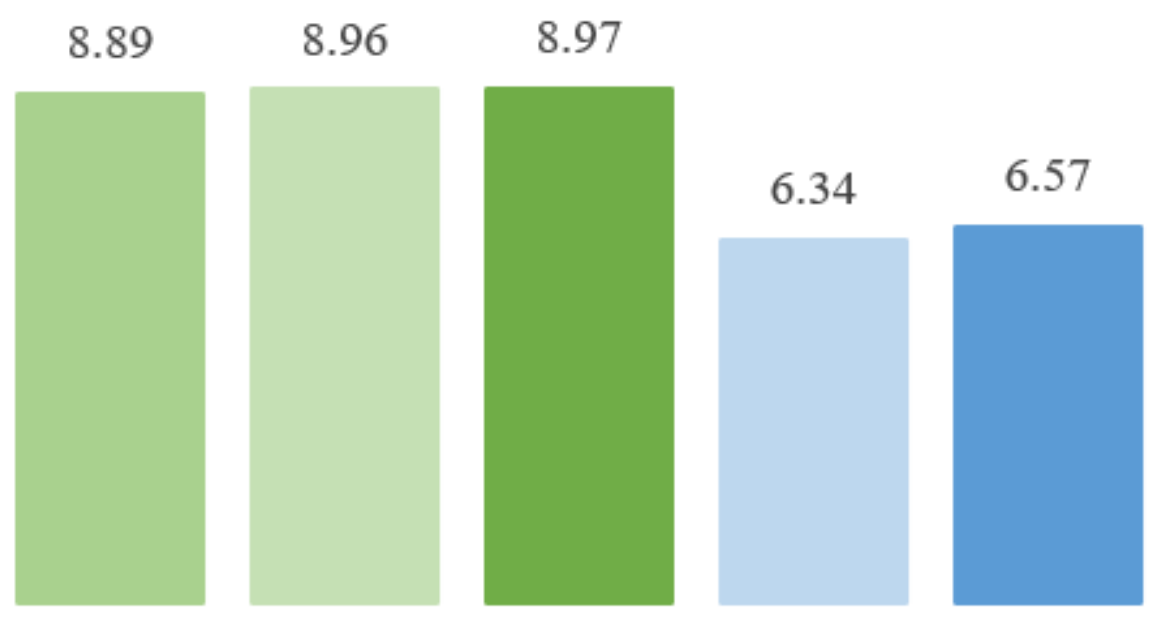

\section{Autoubicación Abascal $\square$ VOX Casado $\square P$}

Fuente: Elaboración propia a partir del Estudio Postelectoral Elecciones Generales en España, noviembre 2019 (EPEGE nov.19).

Pasando a la ubicación en la escala ideológica (gráfico 5), observamos como los valores son muy similares en la propia autoubicación de los votantes y en dónde estos ubican a VOX y al PP. Sin embargo, si profundizamos más en los datos y, teniendo en cuenta que el 10 es el valor más a la derecha y el 0 más a la izquierda, podremos observar como la propia autoubicación de los votantes ha ido evolucionando hacia una posición más a la derecha, pasando de un 6,8 en las elecciones andaluzas de 2018 hasta un 7,36 en las generales de noviembre de 2019. Lo contrario a esto sucede con el PP, pues los encuestados lo sitúan en posiciones más próximas al centro derecha, con un 6,01 en noviembre de 2019. Por último, respecto al partido VOX, de forma similar a lo que ocurrió con sus votantes, su posición en la escala ha ido modificándose hacia una posición más a la derecha. Debemos destacar como sus propios votantes sitúan más a la derecha a su partido que a ellos mismos, habiendo una diferencia de un punto entre ambos en la misma escala. 
Gráfico 5. Escala de ubicación ideológica (0-10)

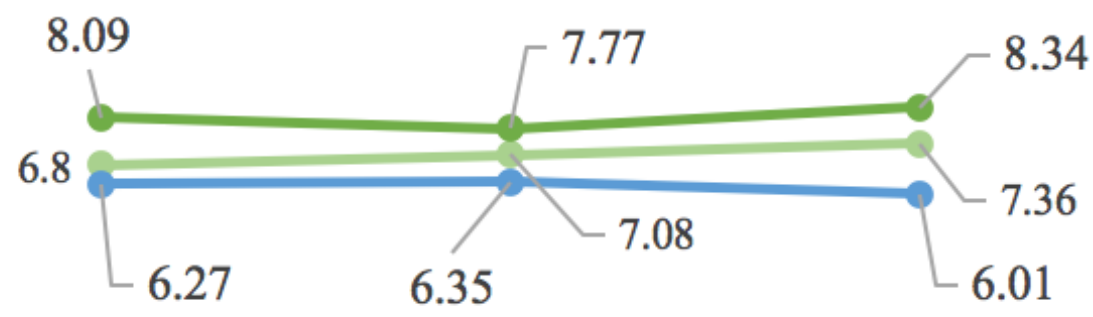

\section{Andalucía $2018 \quad$ Generales abril Generales 2019 noviembre 2019}

\section{—Autoubicación ideológica —Ubicación ideológica PP - -Ubicación ideológica VOX}

Fuente: Elaboración propia a partir del Estudio poselectoral del Equipo de Investigaciones Políticas para las elecciones autonómicas andaluzas 2018, del Estudio Postelectoral Elecciones Generales en España, abril 2019 (EPEGE abr.19) y del Estudio Postelectoral Elecciones Generales en España, noviembre 2019 (EPEGE nov.19).

Gráfico 6. Grado de utilización de redes sociales

\section{- Recibir información política $\quad$ Participación política}

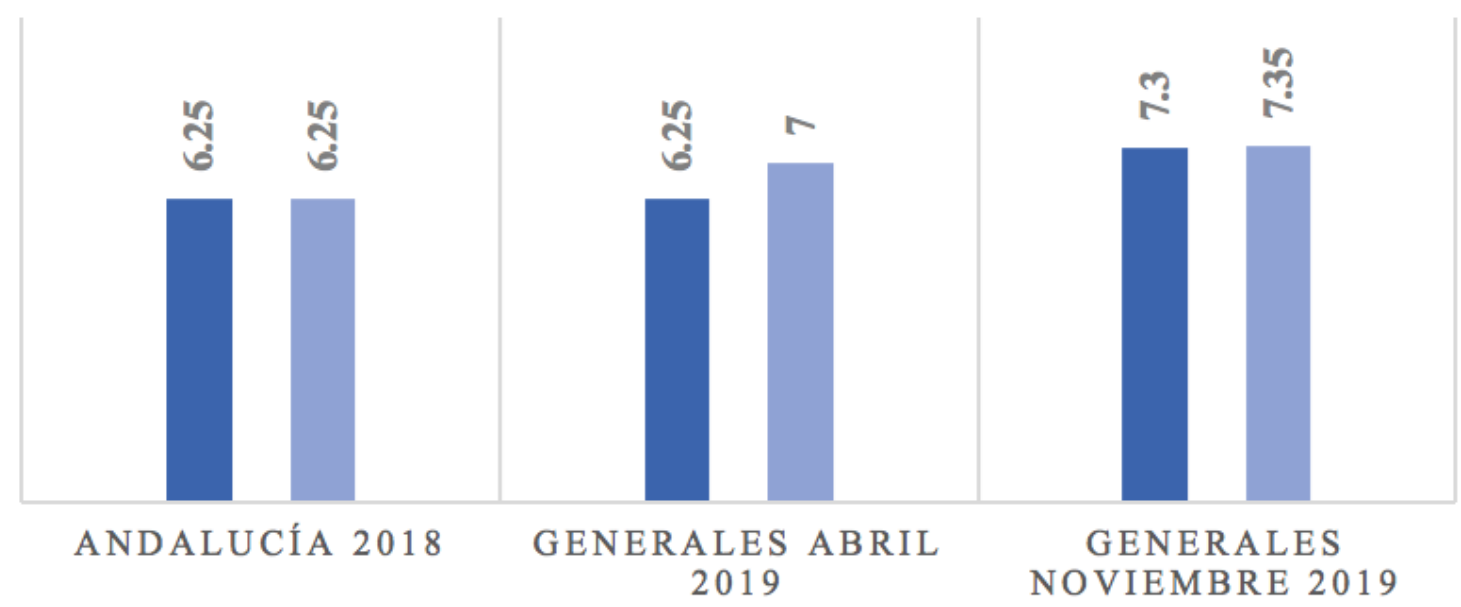

Fuente: Elaboración propia a partir del Estudio poselectoral del Equipo de Investigaciones Políticas para las elecciones autonómicas andaluzas 2018, del Estudio Postelectoral Elecciones Generales en España, abril 2019 (EPEGE abr.19) y del Estudio Postelectoral Elecciones Generales en España, noviembre 2019 (EPEGE nov.19).

Respecto al grado de utilización de las redes sociales, para recibir o participar en política (gráfico 6), observamos como las puntuaciones han evolucionado al alza desde 2018, siendo en los comicios de noviembre dónde se dan los valores más elevados. 


\section{2 ¿Qué lo ha llevado a votar a VOX?}

Pasando a variables de comportamiento de voto, lo primero es conocer de dónde venía el votante de VOX y qué lo motivó a votar a dicho partido.

Empezando por la primera pregunta, las siguientes tablas, nos muestran la transferencia de voto desde las elecciones generales celebradas en 2016. De este modo, comenzando por las elecciones autonómicas de Andalucía, observamos (tabla 5) como el principal nicho de voto de VOX provenía del Partido Popular, pues de los que votaron a VOX en 2018, un 23,6\% habían votado al Partido Popular en las autonómicas de Andalucía de 2015. De los restantes partidos, destacamos un 7,1\% de voto a VOX proveniente de los votantes de Ciudadanos en 2015.

Tabla 5. Transferencia de voto elecciones autonómicas andaluzas 2018 hacia VOX procedentes de otras formaciones

Recuerdo de voto autonómicas Andalucía 2018

\begin{tabular}{|c|c|c|c|c|c|c|}
\hline & & PSOE & PP & AA & C's & VOX \\
\hline $\begin{array}{c}\text { Recuerdo de } \\
\text { voto }\end{array}$ & PSOE & $68,2 \%$ & $7,9 \%$ & $6,6 \%$ & $15,9 \%$ & $1,3 \%$ \\
\hline autonómicas & UP & $0,7 \%$ & $54,3 \%$ & $0,7 \%$ & $20,7 \%$ & $23,6 \%$ \\
\hline $\begin{array}{c}\text { Andalucía } \\
2015\end{array}$ & C's & $5,1 \%$ & & $87,8 \%$ & $6,1 \%$ & \\
\hline & IU & $16 \%$ & $12,5 \%$ & $5,4 \%$ & $69,6 \%$ & $7,1 \%$ \\
\hline
\end{tabular}

Fuente: Elaboración propia a partir del Estudio poselectoral del Equipo de Investigaciones Políticas para las elecciones autonómicas andaluzas 2018, del Estudio Postelectoral Elecciones Generales en España, abril 2019 (EPEGE abr.19) y del Estudio Postelectoral Elecciones Generales en España, noviembre 2019 (EPEGE nov.19).

En el caso de las elecciones generales de abril de 2019 (tabla 6), los votantes de VOX, provenían en un 15,4\% del Partido Popular y en un 12,2\%, de Ciudadanos. Exactamente los mismos nichos de voto que en el caso de las autonómicas andaluzas de 2015. Por último, las elecciones de noviembre (tabla 7), en las cuales ya podemos observar el porcentaje de fidelización de voto a VOX, volvemos a comprobar como los dos sectores de voto de VOX vuelven a ser el PP, en un 13\% y, Ciudadanos, en un $7 \%$. Cierto es que estos porcentajes son menores en comparación con las generales de abril de este mismo año, pero lo cierto es que ya observamos como del total de encuestados que habían dicho haber votado a VOX en noviembre de 2019 , un $82,9 \%$ ya lo había hecho en abril. 
Tabla 6. Transferencia de voto elecciones generales 2016-abril 2019

Recuerdo de voto Generales abril 2019

\begin{tabular}{c|c|c|c|c|c|c|}
\cline { 2 - 8 } Recuerdo & & PSOE & PP & UP & C's & VOX \\
\cline { 2 - 8 } de voto & PSOE & $84,6 \%$ & $2,2 \%$ & $4,8 \%$ & $5,3 \%$ & $0,9 \%$ \\
\hline Generales & PP & $3,2 \%$ & $61,5 \%$ & $0,6 \%$ & $16 \%$ & $15,4 \%$ \\
\hline 2016 & UP & $15,8 \%$ & - & $80 \%$ & $0,8 \%$ & - \\
\hline & C's & $14,4 \%$ & $5,6 \%$ & $1,1 \%$ & $60 \%$ & $12,2 \%$ \\
\hline
\end{tabular}

Fuente: Elaboración propia a partir del Estudio Postelectoral Elecciones Generales en España, abril 2019 (EPEGE abr.19).

Tabla 7. Transferencia de voto elecciones generales abril - noviembre 2019

Recuerdo de voto Generales noviembre 2019

\begin{tabular}{|c|c|c|c|c|c|c|}
\hline \multirow{3}{*}{$\begin{array}{c}\text { Recuerdo } \\
\text { de voto }\end{array}$} & & PSOE & PP & UP & $C^{\prime} s$ & VOX \\
\hline & PSOE & $87,9 \%$ & $0,9 \%$ & $4,3 \%$ & $1,3 \%$ & - \\
\hline & PP & $1,9 \%$ & $78,7 \%$ & - & $2,8 \%$ & $13 \%$ \\
\hline \multirow{3}{*}{$\begin{array}{l}\text { Generales } \\
\text { abril } 2019\end{array}$} & UP & $11,7 \%$ & - & $76,6 \%$ & $0,8 \%$ & - \\
\hline & $C^{\prime} s$ & $10,5 \%$ & $15,1 \%$ & $1,2 \%$ & $55,8 \%$ & $7 \%$ \\
\hline & VOX & - & $12,5 \%$ & $2,4 \%$ & - & $82,9 \%$ \\
\hline
\end{tabular}

Fuente: Elaboración propia a partir del Estudio Postelectoral Elecciones Generales en España, noviembre 2019 (EPEGE nov.19)

Tabla 8. Motivo por el que el entrevistado votó a VOX

Andalucía $2018 \quad$ Generales abril $2019 \quad$ Generales noviembre 2019

\begin{tabular}{|c|c|c|}
\hline Porque es el más capacitado & Porque España necesitaba un & Porque es el más capacitado \\
para gobernar (42,4\%) & gobierno $(33,3 \%)$ & para gobernar $(74,4 \%)$ \\
\hline Para evitar que ganase el & Porque es el más capacitado & Porque España necesitaba un \\
partido adversario $(39,4 \%)$ & para gobernar $(20 \%)$ & gobierno $(9,5 \%)$ \\
\hline
\end{tabular}

Fuente: Elaboración propia a partir del Estudio poselectoral del Equipo de Investigaciones Políticas para las elecciones autonómicas andaluzas 2018, del Estudio Postelectoral Elecciones Generales en España, abril 2019 (EPEGE abr.19) y del Estudio Postelectoral Elecciones Generales en España, noviembre 2019 (EPEGE nov.19).

Respecto al motivo que los ha llevado a votar a VOX, la tabla 7 nos muestra de forma esquemática las diferentes razones para esta decisión electoral. Fácilmente se puede apreciar que la principal y, más repetida razón, es porque lo consideran el partido más capacitado para gobernar. 


\section{$4 \cdot 3$ ¿Cómo es su visión de la política española?}

Acerca de la política en España (gráfico 7), partiendo de una escala en la que 0 es el valor mínimo y 10 el valor máximo, observamos como su interés por la política es bastante elevado, con valores en torno al 6 en las tres elecciones. Sin embargo, a pesar de su interés, su valoración de la situación política ha ido disminuyendo de un 4,98 en 2018, hasta un 1,43 en noviembre de 2019. Una reducción de más de 3 puntos. Por último, respecto a la confianza en la clase política, esta no llega nunca a pasar de un 2,86, siendo este el valor más elevado de entre las tres elecciones. Por tanto, comparando las tres variables, a pesar de que su interés en la política es ciertamente elevado, su visión de esta es nefasta.

Gráfico 7. Visión de la política española (0-10)

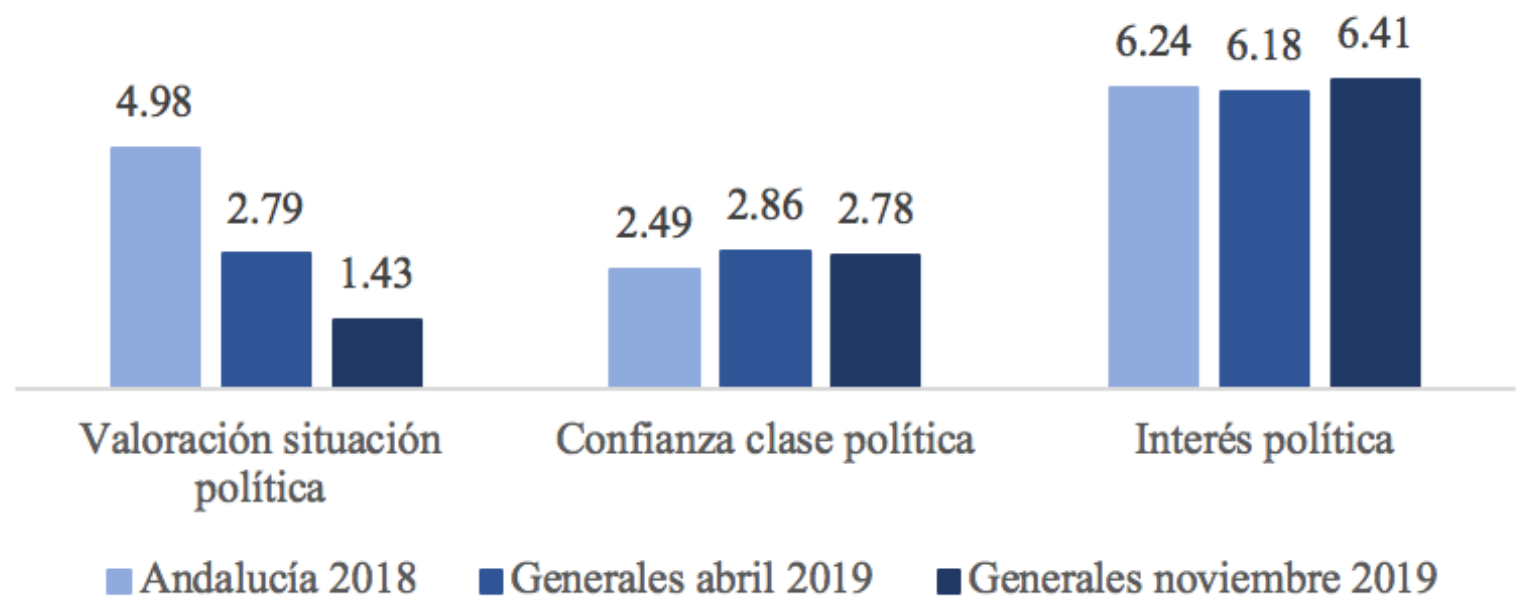

Fuente: Elaboración propia a partir del Estudio poselectoral del Equipo de Investigaciones Políticas para las elecciones autonómicas andaluzas 2018, del Estudio Postelectoral Elecciones Generales en España, abril 2019 (EPEGE abr.19) y del Estudio Postelectoral Elecciones Generales en España, noviembre 2019 (EPEGE nov.19).

\section{4 ¿Una España plural?}

De cara a elaborar un perfil de votante lo más exhaustivo posible, resulta indispensable tener en cuenta la posición de estos votantes respecto a debates actuales en España en cuanto a la multiculturalidad, las libertades y el Estado de Bienestar (gráfico 8).

En primer lugar, sobre si consideran muy positivo o peligroso (siguiendo una escala $0-10$ respectivamente) que en un país convivan personas de diferentes culturas, estos se sitúan en valores próximos al $5 \mathrm{y}$, teniendo en cuenta que el centro en la escala estaría en el 5,5, tienden a aproximarse más a considerarlo peligroso que a entenderlo como algo muy positivo para la sociedad.

En segundo lugar, de cara a una maximización de las libertades, entendiendo 0 como que lo más importante es tener el máximo de libertad aun perdiendo seguridad y, el 10, lo contrario, estos se sitúan en valores superiores al 6 , por lo que se acercan más a priorizar una mayor seguridad frente a una maximización de las libertades. 
Gráfico 8. Visión de una España plural: multiculturalismo, libertades versus seguridad y Estado de Bienestar (0-10)

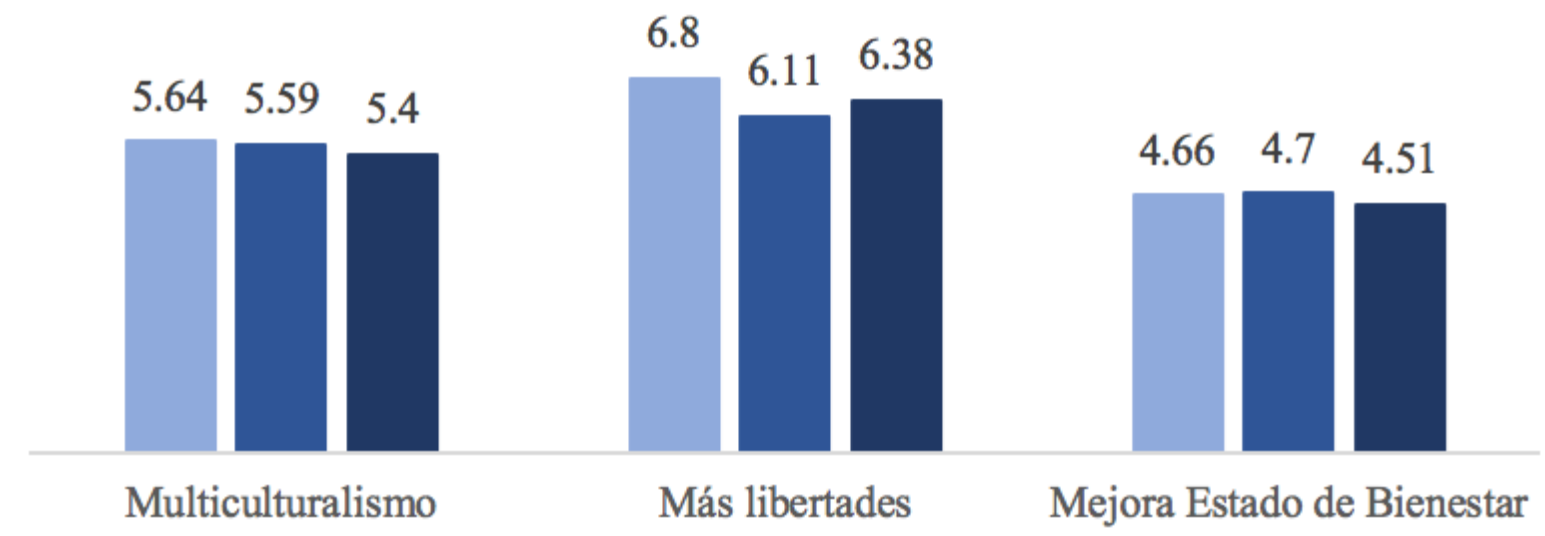

\section{Andalucía 2018 口Generales abril 2019 a Generales noviembre 2019}

Fuente: Elaboración propia a partir del Estudio poselectoral del Equipo de Investigaciones Políticas para las elecciones autonómicas andaluzas 2018, del Estudio Postelectoral Elecciones Generales en España, abril 2019 (EPEGE abr.19) y del Estudio Postelectoral Elecciones Generales en España, noviembre 2019 (EPEGE nov.19).

En tercer lugar, siguiendo esa misma escala, siendo 0 que consideran necesario una mejora del Estado de Bienestar a pesar de una subida de impuestos y 10, pagar menos impuestos aún a pesar del recorte en las políticas de bienestar, estos se encuentran en valores superiores al 4,5. Recordemos que el 5,5 sería el valor central en la escala, por lo que se sitúan más próximos a una mejora del Estado de Bienestar a pesar de una posible subida de impuestos.

\section{5 ¿Cómo ven sus propios votantes a VOX?}

Por último, y para completar la elaboración del perfil de votante de VOX, analizaremos que opinión tienen los votantes de su propio partido y de su líder, Santiago Abascal. Puesto que el nicho de voto de VOX proviene del PP, resulta interesante comparar la valoración de ambos líderes ${ }^{4}$. 
Gráfico 9. Valoración de Santiago Abascal y Pablo Casado (0-10)

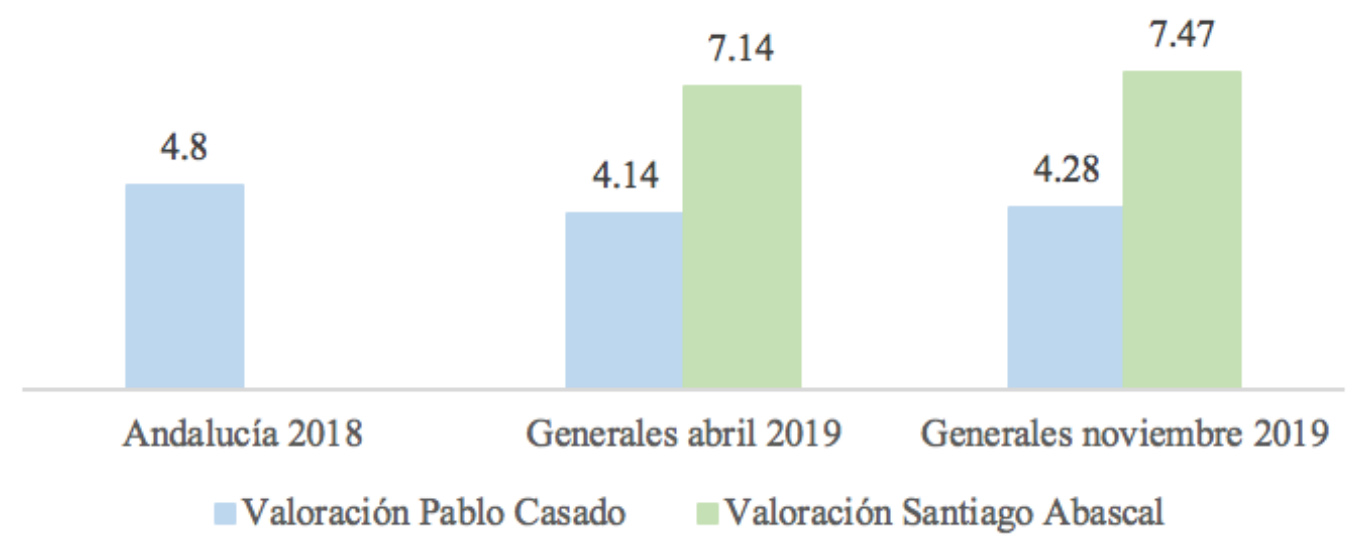

Fuente: Elaboración propia a partir del Estudio poselectoral del Equipo de Investigaciones Políticas para las elecciones autonómicas andaluzas 2018, del Estudio Postelectoral Elecciones Generales en España, abril 2019 (EPEGE abr.19) y del Estudio Postelectoral Elecciones Generales en España, noviembre 2019 (EPEGE nov.19).

A partir del gráfico mostrado en la parte superior (gráfico 9), podemos observar como la valoración de Pablo Casado ha ido disminuyendo, mientras que, en el caso de Abascal, sucede lo contrario, pues no solo le saca más de 3 puntos de diferencia a Pablo Casado, sino que subió en 0,3 su valoración entre los meses de abril y noviembre.

Respecto a lo que Abascal les hace sentir a sus votantes (gráfico 10), observamos como el entusiasmo se ha reducido en más de 2 puntos entre las elecciones de abril y noviembre de 2019 , la esperanza ha aumentado en casi medio punto, el miedo hacia Abascal se ha reducido en casi un punto, el orgullo hacia él ha subido, la preocupación bajó y la tranquilidad aumentó. Por tanto, sienten una mayor esperanza, orgullo y tranquilidad hacia Abascal, a la vez que este les genera menos miedo y menos preocupación, pero también un menor entusiasmo.

Gráfico 10. Emociones de los votantes de VOX hacia Santiago Abascal

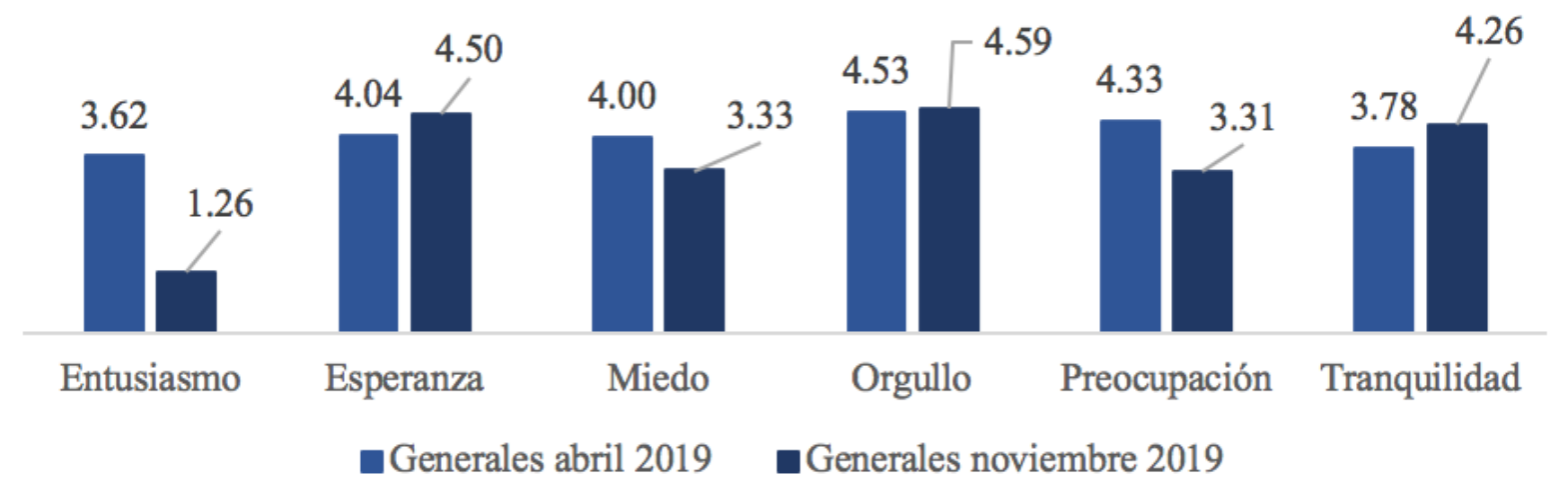

Fuente: Elaboración propia a partir del Estudio Postelectoral Elecciones Generales en España, abril 2019 (EPEGE abr.19) y del Estudio Postelectoral Elecciones Generales en España, noviembre 2019 (EPEGE nov.19).

Dejando a un lado a Abascal y centrándonos en la visión del partido en términos electorales (gráfico 11), podemos destacar cuatro actuaciones. En primer lugar, respecto a el pacto con el PP para permitir el gobierno bipartito entre PP y Ciudadanos en Andalucía, sus votantes lo valoran muy bien, posicionándose en un 8 sobre 10 (siendo 0 muy mal y 10 muy bien). En segundo lugar, sobre la actuación de VOX para la posterior consecución del gobierno de coalición entre PSOE y Unidas 
Podemos (usando la misma escala), sitúan en 5,4 la actuación de VOX, mientras que la del PP, en un 3,12 . Por último, respecto a su grado de acuerdo con el crecimiento electoral de VOX, muestran un alto acuerdo, posicionándose en un 8,83 sobre 10.

Gráfico 11. Visión electoral de VOX

\section{Valoración VOX consecuencia Gobierno coalición}

Valoración PP consecuencia Gobierno coalición

Grado acuerdo crecimiento electoral VOX (noviembre)

Valoración pacto VOX - PP Andalucía

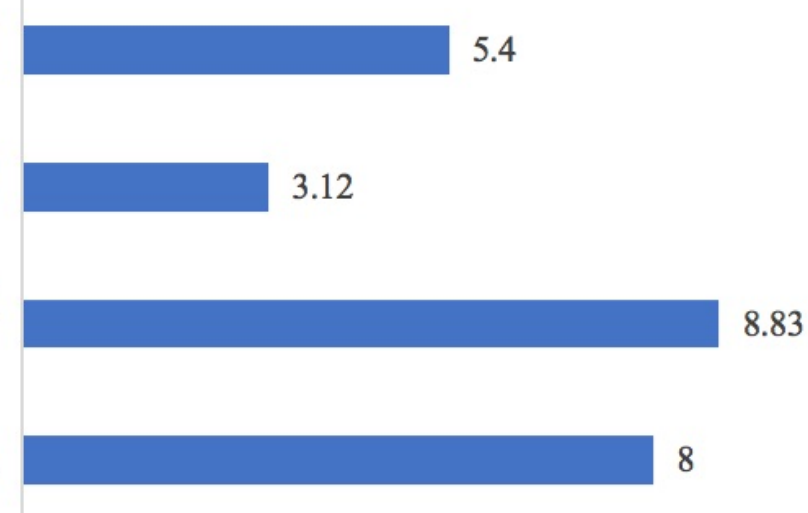

8.83

Fuente: Elaboración propia a partir del Estudio poselectoral del Equipo de Investigaciones Políticas para las elecciones autonómicas andaluzas 2018, del Estudio Postelectoral Elecciones Generales en España, abril 2019 (EPEGE abr.19) y del Estudio Postelectoral Elecciones Generales en España, noviembre 2019 (EPEGE nov.19).

En cuanto a las emociones respecto al partido (gráfico 12), los valores se han mantenido mucho más estables en comparación con los de Santiago Abascal. El entusiasmo se ha reducido en apenas una décima desde 2018 hasta noviembre de 2019, la esperanza ha aumentado en 0,4, y el miedo en 1 punto. El orgullo hacia el partido subió, al igual que la tranquilidad y la preocupación, aunque esta última se redujo en comparación a abril de 2019. Por tanto, la esperanza, la tranquilidad y el orgullo se incrementaron y, la preocupación, el entusiasmo y el miedo se redujeron.

Gráfico 12. Emociones de los votantes de VOX hacia su partido

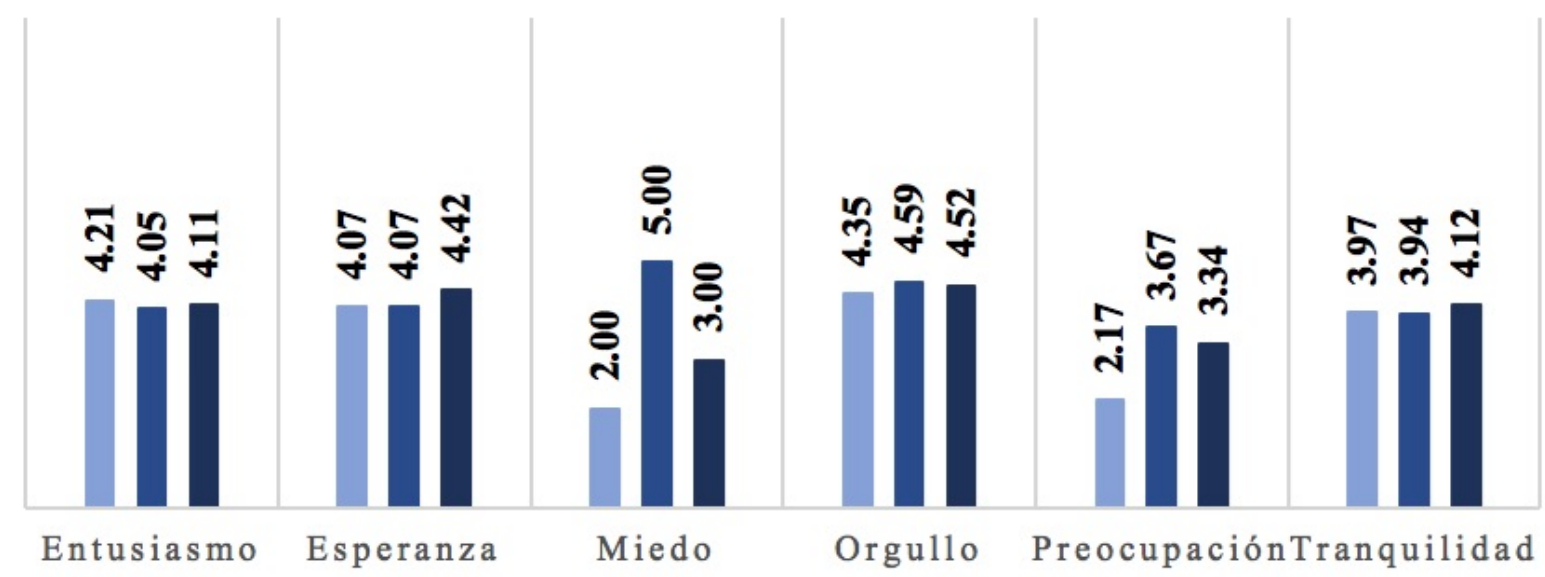

-Andalucía 2018 —Generales abril 2019 a Generales noviembre 2019

Fuente: Elaboración propia a partir del Estudio postelectoral del Equipo de Investigaciones Políticas para las elecciones autonómicas andaluzas 2018, del Estudio Postelectoral Elecciones Generales en España, abril 2019 (EPEGE abr.19) y del Estudio Postelectoral Elecciones Generales en España, noviembre 2019 (EPEGE nov.19). 


\section{COMPOSICIÓN DEL VOTO A VOX}

En el presente apartado del trabajo expondremos la composición del voto a VOX en las elecciones generales de 2019, tanto abril como noviembre, así como en las elecciones autonómicas andaluzas que dan lugar al auge de VOX. Para ello, se realizará para cada elección un análisis de regresión logística binaria con el fin de conocer que elementos resultan significativos a la hora de explicar el voto a este partido (tabla 10 ).

Empezando con las elecciones andaluzas de 2018 (segunda fila de la tabla 10), el elemento con mayor capacidad explicativa para el modelo de las elecciones andaluzas es la simpatía o identificación partidista hacia VOX, con una significatividad máxima. Cuestión llamativa en tanto que el partido apenas contaba con una base sólida y estable de electores y simpatizantes.

En segundo lugar, una valoración negativa hacia Pedro Sánchez también explica el voto hacia VOX. Esto viene a plantear que el voto a VOX en Andalucía, se explica también en clave estatal al ser significativa la valoración del presidente del gobierno español para el voto en Andalucía.

Tabla 9. Nivel de explicación de los modelos

\begin{tabular}{|c|c|c|}
\hline Modelo & $\begin{array}{c}\mathbf{R}^{\mathbf{2}} \text { de } \\
\text { Nagelkerke }\end{array}$ & $\begin{array}{c}\text { 2log } \\
\text { verosimilitud }\end{array}$ \\
\hline Elecciones Andalucía 2018 & $52,4 \%$ & 168,946 \\
\hline Elecciones España 2019 (abril) & $72,3 \%$ & 60,013 \\
\hline Elecciones España 2019 & $73,5 \%$ & 126,888 \\
\hline (noviembre) & & \\
\hline
\end{tabular}

Fuente: Elaboración propia a partir del Estudio poselectoral del Equipo de Investigaciones Políticas para las elecciones autonómicas andaluzas 2018, del Estudio Postelectoral Elecciones Generales en España, abril 2019 (EPEGE abr.19) y del Estudio Postelectoral Elecciones Generales en España, noviembre 2019 (EPEGE nov.19). 
Tabla 10. Resumen explicación voto VOX

\begin{tabular}{|c|c|c|c|}
\hline Variable & $\begin{array}{c}\text { Autonómicas } \\
\text { Andalucía 2018 }\end{array}$ & $\begin{array}{c}\text { Generales 2019 } \\
\text { (abril) }\end{array}$ & $\begin{array}{c}\text { Generales 2019 } \\
\text { (noviembre) }\end{array}$ \\
\hline Simpatía VOX & $3,217^{* * *}$ & $2,734^{* * *}$ & $2,889 * * *$ \\
\hline Valoración Santiago Abascal & $(0,539)$ & $(0,746)$ & $(0,550)$ \\
\hline Valoración Pablo Iglesias & & $0,534 * * *$ & $0,496 * * *$ \\
\hline Valoración Pablo Casado & & $(0,162)$ & $(0,125)$ \\
\hline Valoración Pedro Sánchez & $-0,422 * * *$ & & $-0,277^{* *}$ \\
\hline Auto ubicación ideológica & $(0,131)$ & $-0,597 * *$ & $(, 106)$ \\
\hline Edad & $0,306 *$ & $(0,227)$ & $-0,416 * *$ \\
\hline & $(0,147)$ & & $(0,135)$ \\
\hline
\end{tabular}

Fuente: Elaboración propia a partir del Estudio poselectoral del Equipo de Investigaciones Políticas para las elecciones autonómicas andaluzas 2018, del Estudio Postelectoral Elecciones Generales en España, abril 2019 (EPEGE abr.19) y del Estudio Postelectoral Elecciones Generales en España, noviembre 2019 (EPEGE nov.19).

Respecto a las elecciones generales de abril de 2019 (tercera fila de la tabla 10), el elemento con mayor capacidad explicativa vuelve a ser la identificación o simpatía partidista hacia VOX, en consonancia con el modelo anterior de las elecciones andaluzas. En este sentido, la significatividad es máxima y su coeficiente de Beta, el más elevado del modelo.

Con respecto al liderazgo, resultan significativos dos líderes políticos. En primer lugar, Santiago Abascal, líder de VOX, cuya valoración en positivo aumenta las posibilidades de votar a VOX, es decir, cuanto mejor se valora a Abascal, más probable será el voto al partido. En sentido opuesto, con una significatividad media, observamos la valoración hacia Pablo Casado en sentido negativo, es decir, que cuanto peor se valore a Pablo Casado, mayor será la probabilidad de voto hacia VOX. Este elemento actúa en consonancia a los datos de transferencia de voto (tablas 4,5 y 6 ), ya que como se observa un elevado porcentaje de votantes de VOX provienen del Partido Popular.

En tercer lugar, cuanto más a la derecha se posicione el votante, mayor será la probabilidad de votar a VOX, de nuevo, cuestión razonable debido al posicionamiento ideológico del partido político objeto de nuestro estudio.

Además, aunque con una significatividad mínima, la edad es otro elemento explicativo para el voto a VOX. En este sentido, cuanto más joven sea el ciudadano, mayor será más probable el voto a VOX. Por último, los elementos explicativos en el caso de las elecciones generales de noviembre (cuarta fila de la tabla 10) son los mismos que antes de la repetición electoral. La única novedad reseñable es la incorporación de la valoración de Pablo Iglesias, líder de Unidas Podemos, En este sentido, una valoración negativa de Iglesias aumenta la posibilidad de votar a VOX. 
En resumidas cuentas, podemos afirmar que los elementos significativos para el voto a VOX desde su irrupción en Andalucía hasta las elecciones generales de noviembre de casi un año después (y a la espera de tener datos acerca de las elecciones madrileñas) son la identificación partidista, la valoración de los diferentes líderes políticos en clave estatal y la ubicación ideológica del votante.

\section{CONCLUSIONES}

El análisis realizado en este artículo, utilizando a la vez una aproximación cualitativa y cuantitativa, nos permite llegar a algunas reflexiones de interés respecto a la evolución y el auge de VOX en nuestro país.

En primer lugar, a través del análisis de la Estructura de Oportunidad Política, se puede concluir que VOX se ha servido de un escenario político favorable para construir e impulsar su proyecto de partido de extrema derecha. En concreto, el escenario presentado después del procés y del referéndum del 1 de octubre, pero sobre todo después de la triunfante moción de censura de mayo de 2018, ofreció una oportunidad perfecta a VOX que este partido supo aprovechar.

En segundo lugar, respecto a su perfil sociopolítico, hemos de destacar que el votante medio de VOX es un hombre cuyo nivel de estudios y estatus socioeconómico ha ido aumentado entre 2018 y 2019 (hasta definirse como un trabajador con estudios universitarios y unos ingresos de entre 1.800 y 2.400 euros). Además, políticamente, este votante destaca por su alta desconfianza hacia la clase política, lo que nos puede dar entender su abandono al partido que tradicionalmente votaba (el PP, partido hegemónico de la derecha) y entender a VOX como una opción oportuna y esperanzadora de futuro.

Por último, en cuanto a las variables explicativas clásicas del voto, en el caso de VOX destacan por su importancia tres variables en el conjunto de los modelos presentados: la identificación partidista, el liderazgo político y la ideología. Tres variables que están en consonancia con los hallazgos presentados en otros estudios sobre comportamiento de voto en nuestro país (Lagares y Pereira, 2015; Lagares, 2017; Lagares et al., 2018; Gómez, 2020).

Para finalizar, consideramos que es necesario que de cara a futuras investigaciones que profundicen en la extrema derecha española se tenga en cuenta, además de los resultados que hemos podido adelantar, la posibilidad de ampliar el estudio del caso de VOX; especialmente en lo que a las variables objeto de estudio se refiere, incluyendo entre otras las variables de carácter emocional, las cuales han demostrado ser de gran interés para el estudio del voto (Castro, 2020 y Jaráiz et al., 2020).

\section{BIBLIOGRAFÍA}

ALONSO, S., y ROVIRA, C. (2015). Spain: No Country for the Populist Radical Right? South European Society \& Politics, 20(1), 21-45. https://doi.org/10.1080/13608746.2014.985448

ARROYO, M. (2020). Las causas del apoyo electoral a VOX en España. Política y sociedad (Madrid, Spain), 57(3), 693-717. https://doi.org/10.5209/poso.69206

ASAMBLEA DE MADRID (2021). Resultados definitivos. Recuperado de https://resultados2021.comunidad. madrid/Resultados/Comunidad-Madrid/0/es

ARZHEIMER, K. (2018). Explaining Electoral Support for the Radical Right. En J. Rygren (Ed.), The Oxford Handbook of the Radical Right (pp. 215-246). Oxford University Press. 
BETZ, H. (1994). Radical right-wing populism in Western Europe. Springer.

BETZ, H. (1998). Introduction. En H. Betz, y S. Immerfall (Eds.), The New Politics of the Right: Neo-Populist Parties and Movements in Established Democracies. MacMillan.

CASALS, X. (2000). La ultraderecha española: una presencia ausente (1975-1999). Historia y política, (3), 147-174. Recuperado de http://dialnet.unirioja.es/servlet/oaiart?codigo=295706

CASALS, X. (2017, Noviembre,). ¿Avanza la ultraderecha en España? Cuatro apuntes y una conclusión. Agenda Pública. Recuperado de https://agendapublica.es/avanza-la-ultraderecha-espana-cuatro-apuntes-un a-conclusion/

CASTRO, P. (2020). La construcción emocional de VOX. http://hdl.handle.net/10347/24106

CASTRO, P., y MO, D. (2020). El issue de la inmigración en los votantes de VOX en las Elecciones Generales de noviembre de 2019. Revista De Investigaciones Políticas Y Sociológicas : RIPS, 19(1), 39-58. https:/ /doi.org/10.15304/rips.19.1.6947

FERREIRA, C. (2019). VOX como representante de la derecha radical en España: un estudio sobre su ideología. Revista Española De Ciencia Política, 51, 73-98. https://doi.org/10.21308/recp.51.03

FORD, R., y GOODWIN, M. (2014). Revolt on the right: Explaining support for the radical right in Britain. Routledge.

GAUS, H. (2004). Opgang en verval van extreem-rechts: bijdrage tot de studie van politieke cycli. Academia Press.

GENERALITAT DE CATALUNYA (2021). Resultados definitivos. Recuperado de https://resultados.parlament 2021.cat/resultados/0/catalunya

GOBIERNO VASCO (2020). Resultados electorales: Parlamento vasco $202012 \mathrm{Jul}$. Recuperado de https://ww w.euskadi.eus/ab12aAREWar/resultado/maint

GÓMEZ, R. (2020). Votando al Partido Popular de Galicia: análisis de los componentes del voto a la formación en las elecciones autonómicas (1993-2016). Revista De Investigaciones Políticas Y Sociológicas : RIPS, 19(2), 85-106. https://doi.org/10.15304/rips.19.2.7342

HERNÁNDEZ-CARR, A. (2011). La hora del populismo? Elementos para comprender el «éxito» electoral de Plataforma per Catalunya. Revista de estudios políticos, (153), 47-74. Recuperado de https://dialnet.u nirioja.es/servlet/oaiart?codigo $=3735808$

JARÁIZ, E., LAGARES, N., y PEREIRA, M. (2020). Emociones y decisión de voto. Los componentes de voto en las elecciones generales de 2016 en España. Revista Española de Investigaciones Sociológicas, , 115-135. https://doi.org/10.5477/cis/reis.170.115

JARÁIZ, E., RIVERA, X. M., y CASTRO, P. (2021). Extrema derecha o derecha radical: por qué una nueva semántica. En E. Jaráiz, Á Cazorla y M. Pereira (Eds.), El auge de la extrema derecha en España (pp. 53-88). Tirant lo Blanch.

KITSCHELT, H. (1995). The Radical Right in Western Europe: A Comparative Analysis. Univ. of Michigan Press.

LAGARES, N., y CASTRO, P. (2021). La identificación emocional de la extrema derecha. En E. Jaráiz, Á Cazorla y M. Pereira (Eds.), El Auge de la Extrema Derecha en España (pp. 189-226). Tirant lo Blanch.

LAGARES, N. (2017). Los componentes del voto. In J. M. Rivera, J. Montabes y N. Lagares (Eds.), Cataluña en Proceso.Las elecciones autonómicas de 2015 (pp. 323-345). Tirant lo Blanch.

LAGARES, N., y PEREIRA, M. (2015). De movimiento a partido: el caso de Podemos. Paper presentado en el XII Congreso AECPA.

LAGARES, N., PEREIRA, M., y RIVERA, X. M. (2018). De podemos a las confluencias. En F. J. Llera, M. Baras y J. Montabes (Eds.), Las elecciones generales de 2015 y 2016 (pp. 227-248). Centro de Investigaciones Sociológicas (CIS). 
LÓPEZ, A., y COLOMÉ, G. (2021). La extrema derecha hoy: De Europa a España. En E. Jaráiz, A. Cazorla y M. Pereira (Eds.), El Auge de la Extrema Derecha en España (pp. 21-52). Tirant lo Blanch.

LÓPEZ-LÓPEZ, P., y GONZÁLEZ, S. (2021). Temas, discurso y emociones en la extrema derecha europea. El caso de VOX en España. En E. Jaráiz, Á Cazorla y M. Pereira (Eds.), El Auge de la Extrema Derecha en España (pp. 419-448). Tirant lo Blanch.

LUBBERS, M., GIJSBERTS, M., y SCHEEPERS, P. (2002). Extreme right-wing voting in Western Europe. European Journal of Political Research, 41(3), 345-378.

LUBBERS, M., y SCHEEPERS, P. (2001). Explaining the Trend in Extreme Right-Wing Voting: Germany 19891998. European Sociological Review, 17(4), 431-449. https://doi.org/10.1093/esr/17.4.431

MAGALHÃES, P. C. (2014). Introduction - Financial Crisis, Austerity, and Electoral Politics. Journal of Elections, Public Opinion and Parties, 24(2), 125-133. https://doi.org/10.1080/17457289.2014.887090

MONTABES, J., y CAZORLA, Á. (2021). Condiciones en el surgimiento de la extrema derecha. En E. Jaráiz, Á Cazorla y M. Pereira (Eds.), El Auge de la Extrema Derecha en España (pp. 89-126). Tirant lo Blanch.

MONTERO, J. R., y FERNÁNDEZ ESQUER, C. (2018). Cuatro décadas del sistema electoral español, 1977-2016. Política Y Gobernanza. Revista De Investigaciones Y Análisis Político, (2), 6-46. https://doi.org/10.308 27/polygob.v0i2.7821

MUDDE, C. (2007). Populist radical right parties in Europe (1. publ. ed.). Cambridge Univ. Press. https://do i.org/10.1017/CB09780511492037

MUDDE, C. (2019). The Far Right Today. Polity Press.

NORRIS, P., y INGLEHART, R. (2019). Cultural backlash: Trump, Brexit, and authoritarian populism. Cambridge University Press. https://doi.org/10.1017/9781108595841

PEREIRA, M., JARÁIZ, E., y CAZORLA, Á. (2021). Hacia la cuarta ola de los partidos de extrema derecha: A modo de conclusión. En E. Jaráiz, Á Cazorla y M. Pereira (Eds.), El Auge de la Extrema Derecha en España (pp. 481-501). Tirant lo Blanch.

ROODUIJIN, M. (2018). What unites the voter bases of populist parties? Comparing the electorates of 15 populist parties. European Political Science Review, 10(3), 351-368. https://doi.org/10.1017/S17557 73917000145

RYDGREN, J. (2006). From tax populism to ethnic nationalism: Radical right-wing populism in Sweden. Berghahn Books.

STOCKEMER, D. (2017). The Front National in France: Continuity and Change Under Jean-Marie Le Pen and Marine Le Pen. Springer.

TARROW, S. (1994). Power in Movement: Social Movements and Contencious Politics. Cambridge University Press.

TEREPEROGLOU, E., y TSATSANIS, E. (2016). Realignment under Stress: The July 2015 Referendum and the September Parliamentary Election in Greece. South European Society \& Politics, 21(4), 427-450. https ://doi.org/10.1080/13608746.2016.1208906

TURNBULL-DUGARTE, S. J. (2019). Explaining the end of Spanish exceptionalism and electoral support for VOX. Research \& Politics, 6(2), 1-8. https://doi.org/10.1177/2053168019851680

TURNBULL-DUGARTE, S. J., RAMA, J., y SANTANA, A. (2020). The Baskerville's dog suddenly started barking: voting for VOX in the 2019 Spanish general elections. Political Research Exchange, 2(1), 1-21. https:// doi.org/10.1080/2474736X.2020.1781543

VAN DER BRUG, W., FENNEMA, M., y TILLIE, J. (2005). Why Some Anti-Immigrant Parties Fail and Others Succeed: A Two-Step Model of Aggregate Electoral Support. Comparative Political Studies, 38(5), 537-573. https://doi.org/10.1177/0010414004273928 
WARWICK, P. V. (1998). Disputed cause, disputed effect: The postmaterialist thesis re-examined. Public Opinion Quarterly, , 583-609.

WEAKLIEM, D. L. (2002). The Effects of Education on Political Opinions: An International Study. International Journal of Public Opinion Research, 14(2), 141-157. https://doi.org/10.1093/ijpor/14.2.141

XUNTA DE GALICIA (2020). Eleccións 2020 Parlamento de Galicia: Resultados definitivos. Recuperado de htt ps://resultados2020.xunta.gal/resultados/0/galicia

ZASLOVE, A. (2008). Here to Stay? Populism as a New Party Type. European Review (Chichester, England), 16(3), 319-336. https://doi.org/10.1017/S1062798708000288

Notas

1 Somos conscientes de la importancia que Tarrow, en las siguientes reediciones, adscribe a la violencia política, estableciéndola como una quinta dimensión. No obstante, dado el contexto político español actual, este elemento no nos parece de relevancia, por lo que omitiremos su análisis.

2 En este sentido, es interesante considerar como el (hasta entonces) único caso destacable de la extrema derecha en España (Plataforma per Catalunya) se había desmarcado del eje nacionalista, tanto españolista como catalanista (Hernández-Carr, 2011).

3 Siendo o el mínimo nacionalismo y 10 el máximo nacionalismo.

4 En el estudio postelectoral de 2018, no se incluye la valoración de Santiago Abascal, por lo que nos resulta imposible incluirla en la comparación. 